\title{
Deformable tree models for 2D and 3D branching structures extraction
}

\author{
Julien Mille and Laurent D. Cohen \\ CEREMADE, CNRS UMR 7534, Université Paris Dauphine \\ 75775 Place du Maréchal de Lattre de Tassigny, Paris, France \\ $\{$ mille, cohen $\}$ aceremade.dauphine.fr
}

\begin{abstract}
The proposed model is devoted to the segmentation and reconstruction of branching structures, like vascular trees. We rely on an explicit representation of a deformable tree, where topological relationships between segments are modeled. This allows easy posterior interactions and quantitative analysis, such as measuring diameters or lengths of vessels. Starting from a unique user-provided root point, an initial tree is built with a technique relying on minimal paths. Within the constructed tree, the central curve of each segment and an associated variable radius function evolve in order to satisfy a region homogeneity criterion.
\end{abstract}

\section{Introduction}

The recovery of branching structures, especially vascular trees in MRA, CTA or retinal images, has been an extensively studied subject [2][8][6][14]. In this context, a major concern lies in the extraction of centerlines and thicknesses of significant branches, which make up relevant data for posterior clinical study. For a survey on vessel extraction techniques, the reader may refer to [11]. Specifically, a tree modeling algorithm should be able to handle imaging issues like great variability in length and thickness of segments.

Among various existing techniques, whether they are designed for planar or volumetric images, few approaches provide an explicit modeling of the tree structure, i.e. the topological relationships between segments [2]. A number of vessel extraction methods is devoted to pixel-based segmentation only, without tree extraction. In these methods, pixel classification is performed with respect to a vesselness (or medialness) measure, based on the image Hessian structure [1][7]. On the other hand, many methods dealing with centerlines extraction like [6] or [14] make sequential tracking of segments, in the sense that the whole final tree structure is propagated from one or several seed location(s). Given a search window, points are consecutively added to segments regarding intensity features. The difficulty lies in the implementation of mechanisms dealing with junctions, whether current segment should be split or not. Moreover, topological relationships between segments are not necessarily represented.

We propose a novel deformable tree model, initialized thanks to a technique based on minimal paths [4]. It is slightly related to the work in [8][5][15], as minimal paths intervene in the definition of centerlines. However, our approach differs from those in the sense that the initial tree is built by back-propagation from many endpoints to the user-provided root point, following the geodesic voting method [18]. The obtained structure explicitly represents topological relationships between segments (the parent segment and children are known for a given segment). Then, each segment is endowed with a deformable generalized cylinder defined by a central curve and a radius function, which both evolve with respect to a variational homogeneity criterion [3]. Both 2D and 3D generalized cylinders are presented, namely deformable bands and tubes. We introduce a novel evolution scheme for the entire tree, performed by successive selection and evolution of sequences of segments. We finally present experimental validation on 2D MR angiography images and 3D CT data.

\section{Background}

The minimal path approach by Cohen and Kimmel [4] aims at finding curves of minimal length in a Riemannian space endowed with an isotropic metric. The length of path $\mathcal{C}$ is:

$$
L(\mathcal{C})=\int_{0}^{1} P(\mathcal{C}(s)) d s
$$

where $s$ is the arc length. Potential $P$, which defines the isotropic metric, should be chosen according to the application. In our case, the minimal path is used to define an initial centerline of segment. Its intensity should be almost uniform and close to the intensity of seed point $\mathbf{x}_{0}$, hence 

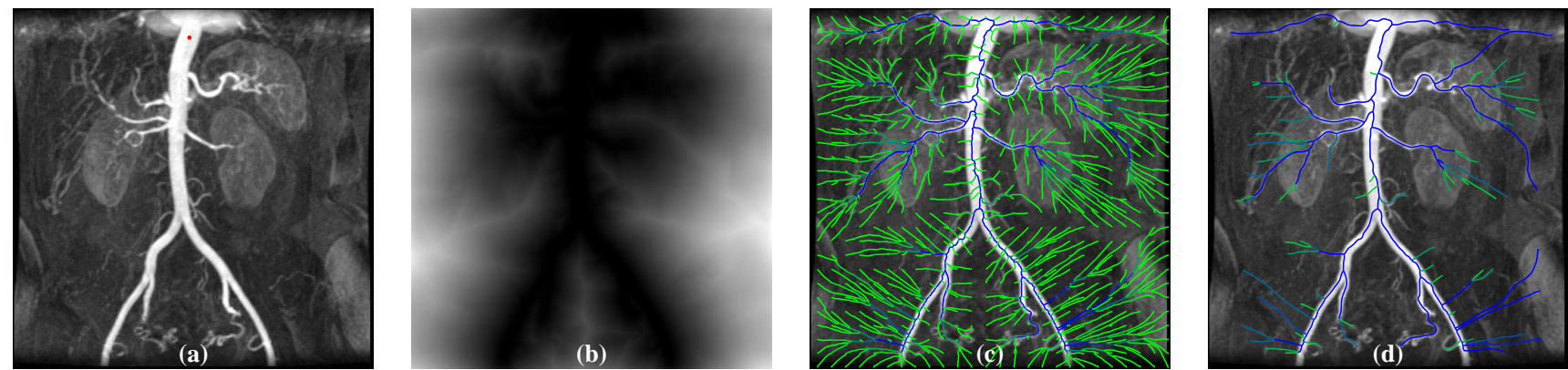

Figure 1. Removing unsignificant segments by vote thresholding : initial image with root point $\mathbf{x}_{\text {root }}$ (a), minimal action map (b), initial tree (c), tree after thresholding on voting score (d)

we choose:

$$
P(\mathbf{x})=w+\left(I(\mathbf{x})-I\left(\mathbf{x}_{0}\right)\right)^{2}
$$

where $I$ is the image intensity and $w$ is a regularizing constant. The minimal path approach determines a global minimum of the energy, given two fixed endpoints $\mathbf{x}_{0}$ and $\mathbf{x}_{1}$. The minimal action map $\mathcal{U}_{0}$, which corresponds to the minimal cost integrated along a path starting at $\mathbf{x}_{0}$ and ending at $\mathbf{x}$, satisfies the Eikonal equation $\|\nabla U\|=P$ and can thus be computed by the Fast Marching method [19]. A geodesic path $\gamma$ linking point $\mathbf{x}_{1}$ to $\mathbf{x}_{0}$ is found by backpropagation on $U$, starting from $\mathbf{x}_{1}$ until $\mathbf{x}_{0}$ is reached: $\partial_{u} \gamma=-\nabla \mathcal{U}_{0}(\gamma)$

The geodesic voting score introduced in [18] is related to path density. Given an action map propagated from one or several seed point(s), the voting score of a point is the number of geodesic paths passing through this point. When several geodesics are extracted, e.g. starting from the borders of the image, they tend to merge at different junction points. The geodesic density and its variation can give significant information on some parts of the structure one wants to extract.

\section{Building the initial tree}

In order to build the initial tree, we extract a large number of geodesic paths. Once the action map starting from root point $\mathbf{x}_{\text {root }}$ is determined (see fig. 1a and 1b), gradient descent is performed from points evenly sampled on a grid. It is assumed that grid step $h$ is small enough so that every branch in the image is partially covered by a path. Since the tree is built by back-propagation starting from endpoints, junctions should be detected. Hence, instead of applying independent back-trackings, we check for the presence of an already traced path at each gradient descent step. During the construction of path $\gamma_{1}$, if the distance between current position $\mathbf{x}_{1}$ and a point $\mathbf{x}_{2}$ in an existing path $\gamma_{2}$ is lower than threshold $\epsilon$ (typically, $\epsilon=1$ ), path $\gamma_{1}$ is stopped and path $\gamma_{2}$ is split at $\mathbf{x}_{2}$. Considering source $\mathbf{x}_{\text {root }}$, the upstream portion of $\gamma_{2}$ becomes the parent segment of $\gamma_{1}$ and the remaining downstream portion of $\gamma_{2}$. Finally, portions of paths between two junction points (or between a junction and an endpoint) become tree segments, which guarantees the acyclic nature of the structure.

As is, one may note that several segments are created without having their endpoints localized in the structure of interest. The geodesic voting score is a relevant clue to assess the significance of segments. In our context, we deal with voting scores of segments, which are computed during the modified gradient descent step described in the previous paragraph. Naturally, the voting score of a segment is the sum of voting scores of his child segments, whereas an endsegment has a unit voting score. Basing ourselves on the fact that significant segments have high voting scores, segment elimination may be performed by thresholding scores. Fig. 1c depicts an initial tree whereas fig. 1d depicts the same tree after removing segments with threshold $s_{\text {vote }}=3$ (color scales from green to blue correspond to score values). As will be justified in section 5, remaining false segments are not critical for the final result.

\section{Deformable generalized cylinders for tubu- lar structures representation}

\subsection{The deformable band}

The deformable band [16], inspired from the ribbon snake model [13], combines aspects of region-based active contours and minimal paths. It is devoted to the recovery of tubular structures. In this context, the segmentation process is constrained by essence, rather than by adding prior shape terms in a general model. The band, depicted in fig. $2 \mathrm{a}$, is defined by open curve $\Gamma$, parameterized by arc length $s \in[0,1]$, and radius function $\mathcal{R}:[0,1] \rightarrow \mathbb{R}^{+}$. Curve $\Gamma$ plays the role of the medial axis. The inner region $R_{\text {in }}$ of width $2 \mathcal{R}$ is bounded by curves $\Gamma_{[\mathcal{R}]}$ and $\Gamma_{[-\mathcal{R}]}$, constructed by translating $\Gamma$ along normal $\mathbf{n}$. Their respec- 


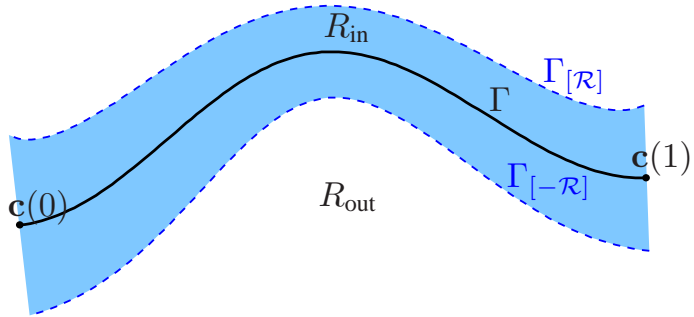

(a)

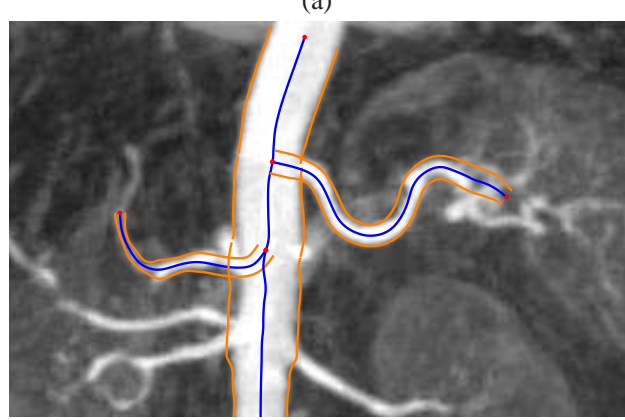

(b)

Figure 2. Deformable band defined by medial curve and thickness (a), representation of the tree by discontinuous bands (b)

tive position vectors are given by:

$$
\begin{aligned}
\mathbf{c}_{[\mathcal{R}]}(s) & =\mathbf{c}(s)+\mathcal{R}(s) \mathbf{n}(s) \\
\mathbf{c}_{[-\mathcal{R}]}(s) & =\mathbf{c}(s)-\mathcal{R}(s) \mathbf{n}(s)
\end{aligned}
$$

The band is endowed with energy functional $E$, weighted sum of the internal energy $E_{\text {smooth }}$ and the external region energy $E_{\text {data }}$ :

$$
E[\Gamma, \mathcal{R}]=\omega E_{\text {smooth }}[\Gamma, \mathcal{R}]+(1-\omega) E_{\text {data }}[\Gamma, \mathcal{R}]
$$

The user-provided coefficient $\omega$, weighting the influence of $E_{\text {smooth }}$ over $E_{\text {data }}$, controls the elastic properties of the deformable band. Since $E$ depends both on $\Gamma$ and $\mathcal{R}$, the band minimizing $E$ should satisfy two coupled EulerLagrange equations:

$$
\frac{\delta E[\Gamma, \mathcal{R}]}{\delta \Gamma}=\mathbf{0} \quad \text { and } \quad \frac{\delta E[\Gamma, \mathcal{R}]}{\delta \mathcal{R}}=0
$$

where the variational derivatives of the energy with respect to the curve and radius vanish. The smoothness energy $E_{\text {smooth }}$ is expressed in terms of curve length - which induces mean curvature motion - and radius first order derivative, as we also seek for a smoothly varying radius:

$$
E_{\text {smooth }}[\Gamma, \mathcal{R}]=\int_{0}^{1}\left\|\mathbf{c}_{s}\right\|+\mathcal{R}_{s}^{2} d s
$$

Since the structure of interest should satisfy an intensity homogeneity criterion, the data term is as follows:

$$
E_{\text {data }}[\Gamma, \mathcal{R}]=\int_{R_{\text {in }}} g_{\text {in }}(\mathbf{x}) d \mathbf{x}+\int_{R_{\text {out }}} g_{\text {out }}(\mathbf{x}) d \mathbf{x}
$$

where region descriptors $g_{\text {in }}$ and $g_{\text {out }}$ increase with respect to intensity inhomogeneity. The band integral over region $R_{\text {in }}$ is explicitly expressed in terms of $\Gamma$ and $\mathcal{R}$ using a mathematical derivation based on Green's theorem. Such derivation enables to calculate variations of $E_{\text {data }}$ and solve associated Euler-Lagrange equations. The variational derivatives of $E_{\text {data }}$ are:

$$
\begin{gathered}
\frac{\delta E_{\text {data }}}{\delta \Gamma} \approx \quad\left[(1-\mathcal{R} \kappa)\left(g_{\text {in }}\left(\mathbf{c}_{[\mathcal{R}]}\right)-g_{\text {out }}\left(\mathbf{c}_{[\mathcal{R}]}\right)\right)\right. \\
\left.-(1+\mathcal{R} \kappa)\left(g_{\text {in }}\left(\mathbf{c}_{[-\mathcal{R}]}\right)-g_{\text {out }}\left(\mathbf{c}_{[-\mathcal{R}]}\right)\right)\right] \mathbf{n} \\
\frac{\delta E_{\text {data }}}{\delta \mathcal{R}} \approx \begin{array}{c}
(1-\mathcal{R} \kappa)\left(g_{\text {in }}\left(\mathbf{c}_{[\mathcal{R}]}\right)-g_{\text {out }}\left(\mathbf{c}_{[\mathcal{R}]}\right)\right) \\
+(1+\mathcal{R} \kappa)\left(g_{\text {in }}\left(\mathbf{c}_{[-\mathcal{R}]}\right)-g_{\text {out }}\left(\mathbf{c}_{[-\mathcal{R}]}\right)\right)
\end{array}
\end{gathered}
$$

We use $g_{\text {in }}(\mathbf{x})=\left(I(\mathbf{x})-\mu_{\text {tree }}\right)^{2}$ and $g_{\text {out }}(\mathbf{x})=(I(\mathbf{x})-$ $\left.\mu_{\text {out }}\right)^{2}$, where $\mu_{\text {tree }}$ and $\mu_{\text {out }}$ are the average intensities inside and outside the entire tree structure, respectively. Note that these functions are related to the potential defined in eq. 2. The band is implemented as a polygonal line of vertices $\mathbf{p}_{i}$ with associated radii $\mathcal{R}_{i}$. Vertex positions and corresponding radii are updated by gradient descent of the Euler-Lagrange equations:

$$
\begin{aligned}
\mathbf{p}_{i}^{(t+1)} & =\mathbf{p}_{i}^{(t)}-\Delta t \delta E /\left.\delta \Gamma\right|_{\mathbf{c}=\mathbf{p}_{i}} \\
\mathcal{R}_{i}^{(t+1)} & =\mathcal{R}_{i}^{(t)}-\Delta t \delta E /\left.\delta \mathcal{R}\right|_{\mathcal{R}=\mathcal{R}_{i}}
\end{aligned}
$$

To maintain sufficient vertex density, the curve is resampled after each deformation step, i.e. after the previous scheme has been applied on all vertices. The initial formulation of the deformable band [16] defines static endpoints. In our context, endpoints are made variable to allow local growing or retraction with respect to region homogeneity. The following force is added to the overall displacement of a variable endpoint:

$$
\mathbf{f}_{\text {data }}\left(\mathbf{p}_{i}\right)=\left[g_{\text {out }}\left(\mathbf{p}_{i}+\mathcal{R}_{i} \mathbf{t}_{i}\right)-g_{\text {in }}\left(\mathbf{p}_{i}+\mathcal{R}_{i} \mathbf{t}_{i}\right)\right] \mathbf{t}_{i}
$$

where $\mathbf{t}_{i}$ is the "outward" unit tangent vector, e.g. in the direction $\mathbf{p}_{1}-\mathbf{p}_{2}$ for endpoint $\mathbf{p}_{1}$.

\subsection{Extension to 3D: the deformable tube}

We present an intuitive 3D extension of the deformable band model described in section 4.1. Let $\phi:[0,1] \rightarrow \mathbb{R}^{3}$ be the curved axis, parameterized by arc-length $s$, and $\mathcal{R}$ : $[0,1] \times[0,2 \pi] \rightarrow \mathbb{R}^{+}$the radius function. Let $\mathbf{T}, \mathbf{N}$ and $\mathbf{B}$ be the tangent, normal and binormal vectors of curve $\phi$. Frame $\{\mathbf{T}, \mathbf{N}, \mathbf{B}\}$ defines an orthogonal coordinate system sweeping along the curve. It is used to build varying crosssections orthogonal to the curve's local direction. Hence, the surface position vector is defined as follows:

$$
\mathbf{s}(s, v)=\phi(s)+\mathcal{R}(s, v)(\cos v \mathbf{N}+\sin v \mathbf{B})
$$

where parameters $s$ and $v$ sweep along and around the cylinder, respectively. As stated in [17], defining the surface in 
this way yields an undesirable twisting effect on the cylinder. Actually, we compute corrected normal and binormal vectors (denoted with ${ }^{*}$ ) following [20]:

$$
\begin{aligned}
\mathbf{N}^{*}(s+\Delta s) & =\frac{\mathbf{N}^{*}(s)-\left\langle\mathbf{N}^{*}(s), \mathbf{T}(s+\Delta s)\right\rangle \mathbf{T}(s+\Delta s)}{\left\|\mathbf{N}^{*}(s)-\left\langle\mathbf{N}^{*}(s), \mathbf{T}(s+\Delta s)\right\rangle \mathbf{T}(s+\Delta s)\right\|} \\
\mathbf{B}^{*}(s+\Delta s) & =\mathbf{T}^{*}(s+\Delta s) \times \mathbf{N}^{*}(s+\Delta s)
\end{aligned}
$$

which basically consists in projecting previous normal and binormal vectors on the current orthogonal plane. The generalized cylinder is endowed with a similar energy as in eq. 4. Equally, a minimizer of $E$ satisfies eq. 5. To express the regularization term, we rely on the reasonable assumption that a smooth axis curve and a smoothly varying radius will yield a smooth surface:

$$
E_{\text {smooth }}[\phi, \mathcal{R}]=\int_{0}^{1}\left\|\phi_{s}\right\| d s+\int_{0}^{1} \int_{0}^{2 \pi} \mathcal{R}_{s}^{2}+\mathcal{R}_{v}^{2} d v d s
$$

The first variations of the smoothness term are:

$$
\begin{aligned}
\frac{\delta E_{\text {smooth }}}{\delta \phi} & =-\left\|\phi_{s}\right\| \kappa \mathbf{N} \\
\frac{\delta E_{\text {smooth }}}{\delta \mathcal{R}} & =-2\left(\mathcal{R}_{s s}+\mathcal{R}_{v v}\right)
\end{aligned}
$$

where $\kappa$ is the curvature of $\phi$. Since the structure of interest should satisfy an intensity homogeneity criterion, the data term is as follows:

$$
E_{\text {data }}[\phi, \mathcal{R}]=\int_{R_{\text {in }}} g_{\text {in }}(\mathbf{x}) d \mathbf{x}+\int_{R_{\text {out }}} g_{\text {out }}(\mathbf{x}) d \mathbf{x}
$$

where region descriptors $g_{\text {in }}$ and $g_{\text {out }}$ increase with respect to intensity inhomogeneity. To obtain a suitable expression of the data term for derivation and implementation, we rely on the divergence theorem. Since surface $\Gamma$ is piecewisedifferentiable, the volume integral of a $\mathbb{R}^{3} \rightarrow \mathbb{R}$ function $f$ on region $R_{\text {in }}$ may be rewriten:

$$
\begin{gathered}
\int_{R_{\mathrm{in}}} f(\mathbf{x}) d \mathbf{x}=-\int_{0}^{1} \int_{0}^{2 \pi}\left\langle\mathbf{F}(\mathbf{s}), \mathbf{s}_{s} \times \mathbf{s}_{v}\right\rangle d v d s \\
-\int_{0}^{2 \pi} \int_{0}^{1}\left\langle\mathbf{F}\left(\mathbf{s}_{1}\right), \mathbf{s}_{1 u} \times \mathbf{s}_{1 v}\right\rangle+\left\langle\mathbf{F}\left(\mathbf{s}_{2}\right), \mathbf{s}_{2 u} \times \mathbf{s}_{2 v}\right\rangle d u d v
\end{gathered}
$$

where vector field $\mathbf{F}$ is chosen such that $\operatorname{div} \mathbf{F}=f$, whereas $\mathbf{s}_{1}$ and $\mathbf{s}_{2}$ are parameterizations of the end cross sections (at $s=0$ and $s=1$, respectively). Admitting that $\mathbf{N}$ and $\mathbf{B}$ can be computed at arc-lengths 0 and 1, we have:

$$
\begin{aligned}
& \mathbf{s}_{1}(u, v)=\phi(0)+u \mathcal{R}(0, v)\left(\cos v \mathbf{N}^{*}(0)+\sin v \mathbf{B}^{*}(0)\right) \\
& \mathbf{s}_{2}(u, v)=\phi(1)+u \mathcal{R}(1, v)\left(\cos v \mathbf{N}^{*}(1)+\sin v \mathbf{B}^{*}(1)\right)
\end{aligned}
$$

It can be shown that the variational derivative of the surface integral in eq. 11 with respect to radius is:

$$
\frac{\delta}{\delta \mathcal{R}}\left\{\int_{R_{\text {in }}} f(\mathbf{x}) d \mathbf{x}\right\}=\mathcal{R}(1-\mathcal{R} \kappa \cos v) f(\mathbf{s})
$$

where $\kappa$ is the curvature of $\phi$. One may easily deduce the first variation of the data term with respect to radius:

$$
\frac{\delta E_{\text {data }}}{\delta \mathcal{R}}=\mathcal{R}(1-\mathcal{R} \kappa \cos v)\left(g_{\text {in }}(\mathbf{s})-g_{\text {out }}(\mathbf{s})\right)
$$

which is actually divided by $\mathcal{R}$ when implemented in the gradient descent, so that an increase of radius does not yield an increase in its variation. The tube is implemented as a polygonal line of $n$ vertices $\mathbf{p}_{i}$, each vertex being endowed with $m$ angular positions with associated radii $\mathcal{R}_{i j}$. Regarding our implementation, it is not necessary to calculate the first variation of $E_{\text {data }}$ with respect to the axis. Indeed, since the axis should remain in the center of the tube, vertex coordinates are computed as centroids of angular positions when all radii have been updated. This is summarized by the following scheme:

$$
\begin{aligned}
& \mathcal{R}_{i j}^{(t+1)}=\mathcal{R}_{i j}^{(t)}-\Delta t \delta E /\left.\delta \mathcal{R}\right|_{\mathcal{R}=\mathcal{R}_{i j}} \\
& \mathbf{p}_{i}^{(t+1)}=\mathbf{p}_{i}^{(t)} \\
& \quad+\frac{1}{m} \sum_{j=1}^{m} \mathcal{R}_{i j}^{(t+1)}\left(\cos \left(\frac{2 \pi j}{m}\right) \mathbf{N}_{i}^{(t)}+\sin \left(\frac{2 \pi j}{m}\right) \mathbf{B}_{i}^{(t)}\right) \\
& \mathbf{p}_{i}^{(t+2)}=\mathbf{p}_{i}^{(t+1)}+\Delta t \delta E_{\text {smooth }} /\left.\delta \phi\right|_{\phi=\mathbf{p}_{i}}
\end{aligned}
$$

where $t+2$ actually corresponds to the smoothing step. As in the $2 \mathrm{D}$ case, endpoints are made dynamic by adding an extra data force, which is computed in a similar way than in eq. 9.

\section{Evolving the tree structure}

\subsection{Evolution scheme}

We now describe the tree evolution scheme, relying on the deformable cylinder models and the geodesic tree generated in section 3. A hierarchical structure of bands, which is referred to as deformable tree, is built by assigning a deformable cylinder model, in $2 \mathrm{D}$ or $3 \mathrm{D}$, to each segment. The segment curve naturally becomes the medial curve of the cylinder, whereas the radius is set to a small initial value $\mathcal{R}^{(0)}$. This generates a discontinuous representation - depicted in fig. $2 \mathrm{~b}$ - which will be subsequently smoothed. Segments are endowed with two status variables denoted ACTIVE and STABLE, indicating respectively if segment should deform at the current iteration and if they reached their final states. Initially, all ACTIVE and STABLE values are disabled. Evolution of the tree is achieved by repeating the following steps, until all segments reach stability:

- Select the connex sequence of segments of maximal euclidean length, such that the last segment is not STABLE

- Mark all segments in the sequence as ACTIVE

- Evolve ACTIVE segments according to gradient descent of eq. 8 
- Update average intensities $\mu_{\text {tree }}$ and $\mu_{\text {out }}$

- Mark all segments in the sequence as STABLE and disable their ACTIVE status. Remove unsignificant segments

Deformable modeling enables to grow partial segments, or retract false segments. The additional force in eq. 9 is only applied on free endpoints of end segments. In the last step, unsignificant segments are determined according to two criteria. The first one deals with the proportion of segment overlapped by other segments. If this proportion is greater than a specified threshold $s_{\text {overlap, }}$, the segment is removed. The second criterion is the one used in [8], stating that the length of a segment should be at least twice greater than its average width.

\subsection{Boundary refinement}

For a given segment, the boundary is easily determined from the central curve and thickness data varying along this curve. However, if cylinders are considered independently, this leads to a discontinuous boundary of the tree structure. To obtain a continuous representation, the boundary may be refined after the deformation using an active contour or surface. For the $2 \mathrm{D}$ tree, we build a closed curve $\mathcal{C}$ by scanning the tree according to a depth-first algorithm. In each segment, the central curve is visited forward (from $\mathbf{c}(0)$ to $\mathbf{c}(1)$, to scan $\mathbf{c}+\mathcal{R} \mathbf{n}$ points) and backward (for $\mathbf{c}-\mathcal{R} \mathbf{n}$ points).

Since topology preservation of the boundary is needed, an explicit implementation of the active contour is used. We use a similar data term as in eq. 7, which can be thought of as an explicit implementation of the Chan \& Vese region energy [3]. The geometry of the boundary should remain tubular to be consistent with the set of tree centerlines, which in this case are related to the shape skeleton. In addition to the region energy and the usual smoothness term, we add a tubularity-maintaining energy, which relates points on $\mathcal{C}$ to their corresponding points on the tree centerline:

$$
E_{\text {tubular }}[\mathcal{C}]=\int_{0}^{1}(1-\langle\mathcal{C}(v)-\mathbf{c}(s), \pm \mathbf{n}(s)\rangle)^{2} d v
$$

where $\mathbf{c}(s)$ is the tree point associated to boundary point $\mathcal{C}(v)$. The sign applied to the normal depends on the scanning step of the centerline, during which boundary point $\mathcal{C}(v)$ was previously created. Fig. 3 represents the $2 \mathrm{D}$ tree and the smoothed boundary curve at different evolution steps. For the 3D tree model, we implement the boundary surface as a triangulated mesh [12]. First, the insides of tubes are voxelized using a polyhedron filling algorithm [10]. Then, the boundary of the obtained discrete volume is triangulated by means of a Marching cube-like method [9]. In the future, a direct transformation from the set of cylindrical surfaces to the triangulated boudnary surface will be considered. This will imply to deal with surface intersections of connected cylinders.

\section{Experiments}

The 2D deformable tree model is tested on a set of MR angiography images. Fig. 5 depicts 4 of them. In all experiments, the initial seed point is provided at a coherent root location. In the last row, the seed correspond to the location where the boundary curve is cut straight (the above remaining tree is manually deleted). Average image size is $512 \times 512$. The grid step $h$ is set to 10 pixels, which turns out to be sufficient for covering, at least partially, every branch of the structure. Threshold $s_{\text {vote }}$ is chosen in order not to remove significant segments. Remaining false segments are not critical regarding the final segmentation result, but only on the computational load. Indeed, the evolution phase needs more iterations as false segments are longer and more numerous. Threshold values of 3 or 4 for $s_{\text {vote }}$ lead to proper false segment removal. During the evolution phase, to delete small segments partially overlapped by other segments, the threshold $s_{\text {overlap }}$ is set to 0.75 . The initial inner thickness $B^{(0)}$ is typically set to 4 pixels. As regards smoothness weight $\omega$, values ranging from 0.4 to 0.7 are sufficient to maintain centerlines smooth. The same set of parameters is suitable for all experiments, in 2D and 3D. Fig. 6 and 7 depict results obtained with the $3 \mathrm{D}$ tree method applied on a CT volume data. Fig. 4 represents a slice of the CT data, with centerlines and surface positions of two segments (aorta and superior mesenteric artery).

With a C++ implementation running on an Intel Core 2 Duo 2.2GHz PC (4Gb RAM), computational costs yielded by the $2 \mathrm{D}$ model are as follows: $0.9 \mathrm{~s}$ for the construction of the initial tree and vote thresholding, $\approx 6 \mathrm{~s}$ for tree evolution and $0.25 \mathrm{~s}$ for building and evolution of the refined boundary. On the $256 \times 256 \times 256$ volume image shown in fig. 6 , our $3 \mathrm{D}$ approach took $8.3 \mathrm{~s}$ for the construction of the initial tree and vote thresholding, $\approx 15 \mathrm{~s}$ for tree evolution and $4.5 \mathrm{~s}$ for building and evolution of the refined boundary surface.

According to visual inspection, we believe the reconstruction results to be promising. Initial curves provided by the minimal path approach are sinuous and do not match vessel centerlines. This phenomenon is corrected during tree evolution, as gradient descent of the region criterion makes curve naturally locate on actual centerlines. The 3D tree of the aorta is validated with respect to the locations of arteries (see right part of fig. 6). To keep a critical eye on our approach, we observe that tiny vessels, which appear darker than thick vessels, are removed from the structure because 

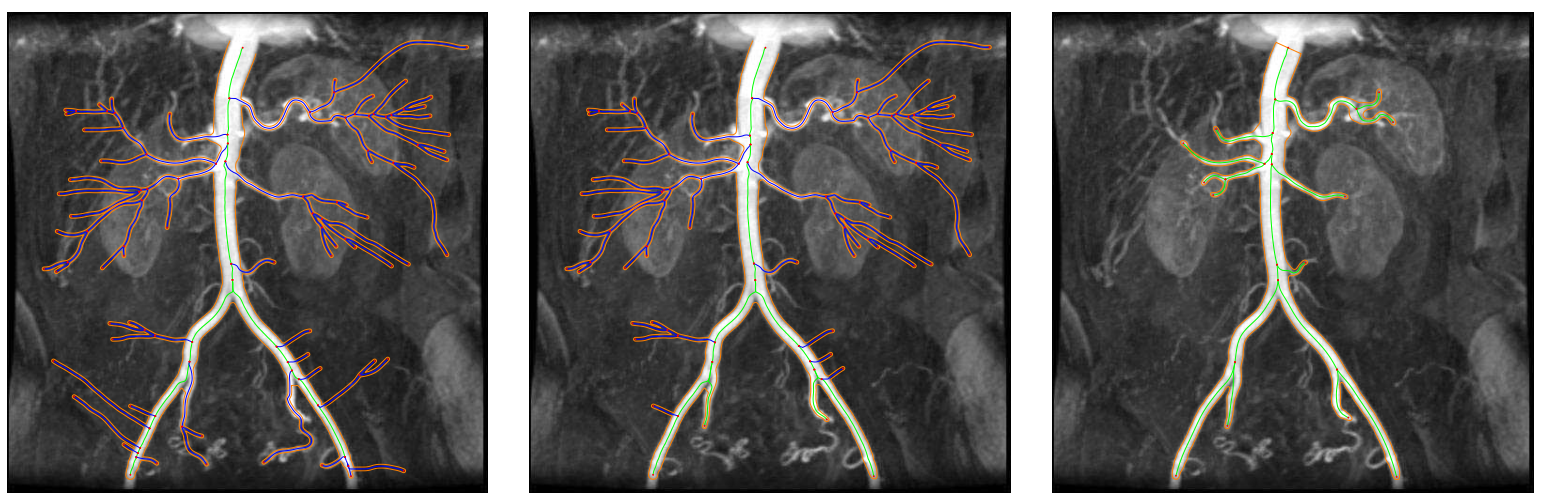

Figure 3. Intermediate steps (left, center) and final step (right) of tree evolution. Colors correspond to NON STABLE, STABLE and ACTIVE segments

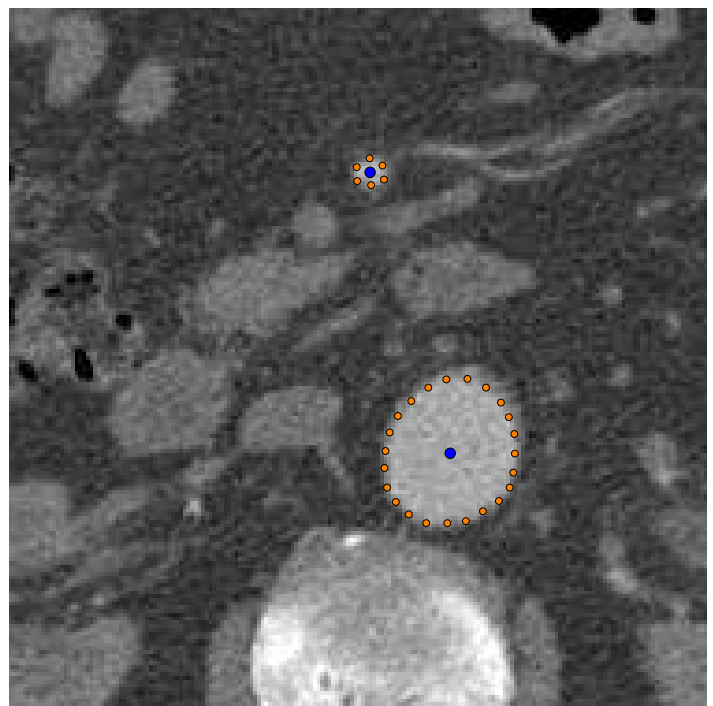

Figure 4. Slice of the 3D CT image, with centerlines and surface positions of two segments: aorta (bottom) and superior mesenteric artery (top)

of the global homogeneity criterion (see eq. 7). One may note that this is not necessarily problematic, depending on ground truth, whether these vessels have to be included or not. However, increased sensivity to small vessels may be achieved by defined a more local region energy. Moreover, in some parts, improvements could be done on the location of intersections, which may appear more upstream than their actual position.

\section{Conclusion and future work}

We described an explicit deformable tree, holding relationships between segments, for the extraction of branching structures. A thresholding technique was applied on the tree in order to remove unsignificant segments, with respect to the geodesic voting score. Deformation of the entire tree was performed by evolving segment cylinders towards a minimum of an energy functional. The evolution method enabled to retract or grow segments in order to satisfy intensity homogeneity inside and outside the tree structure. Our approach was validated on 2D MRI and 3D CT data.

As a future investigation, validation on a large database holding expert segmentations will follow. Future work will focus on the formulation of local region terms handling intensity variations along branches, which we believe to be valuable for extracting thin and low-contrasted vessels. We may also consider an improvement on the selection of initial segment endpoints, which could be done by studying particular differential quantities on the action map.

\section{References}

[1] S. Aylward and E. Bullitt. Initialization, noise, singularities, and scale in height ridge traversal for tubular object centerline extraction. IEEE Transactions on Medical Imaging, 21(2):61-75, 2002.

[2] T. Bülow, C. Lorenz, and S. Renisch. A general framework for tree segmentation and reconstruction from medical volume data. In MICCAI, pages 533-540, Saint-Malo, France, 2004.

[3] T. Chan and L. Vese. Active contours without edges. IEEE Transactions on Image Processing, 10(2):266277, 2001.

[4] L. Cohen and R. Kimmel. Global minimum for active contour models: a minimal path approach. International Journal of Computer Vision, 24(1):57-78, 1997.

[5] T. Deschamps and L. Cohen. Fast extraction of tubular and tree 3D surfaces with front propagation methods. In ICPR, Quebec, Canada, 2002.

[6] N. Flasque, M. Desvignes, J.-M. Constans, and M. Revenu. Acquisition, segmentation and tracking 

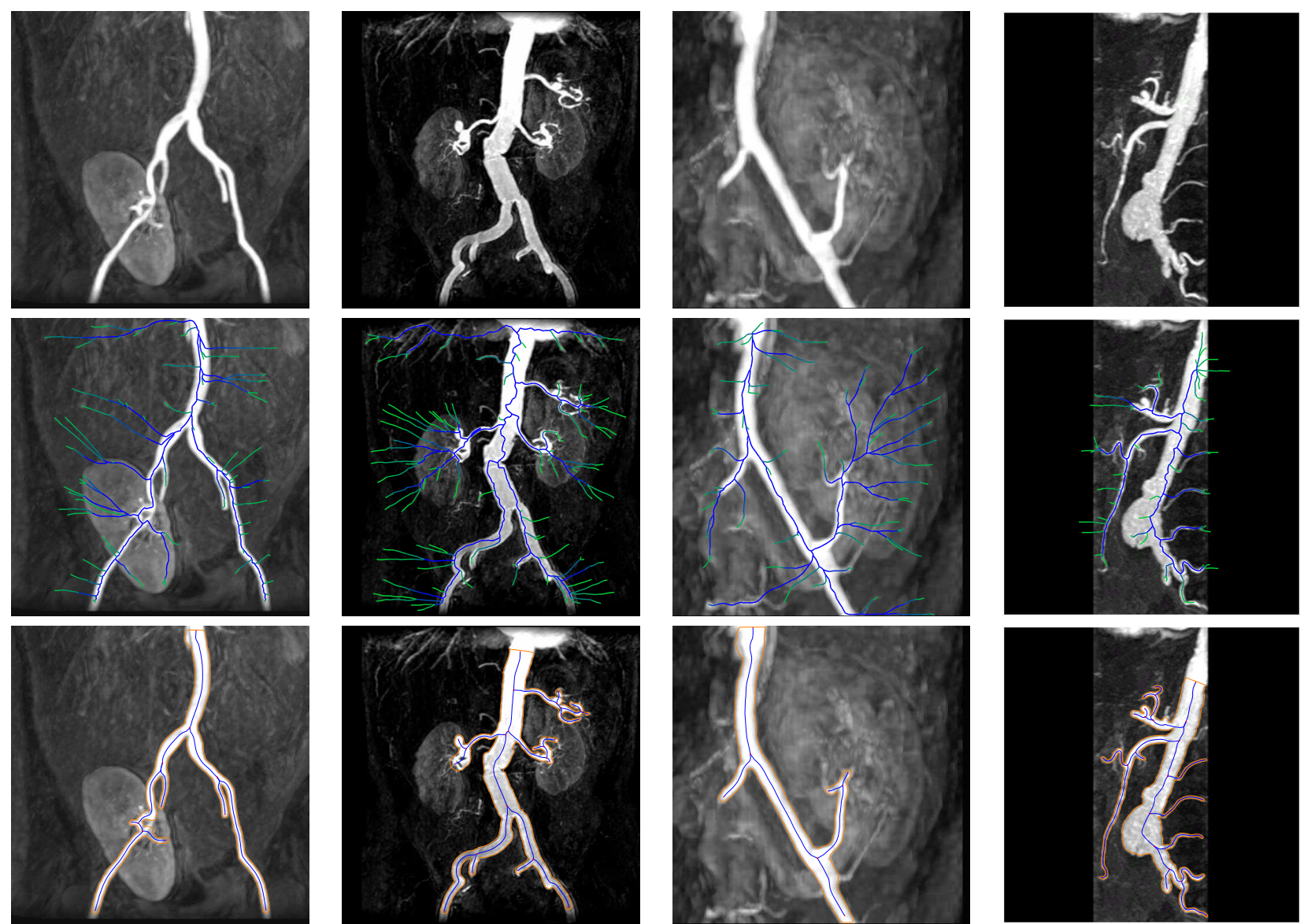

Figure 5. Original images (top row), trees after thresholding on voting score (middle row), final trees with boundary curve (bottom row)
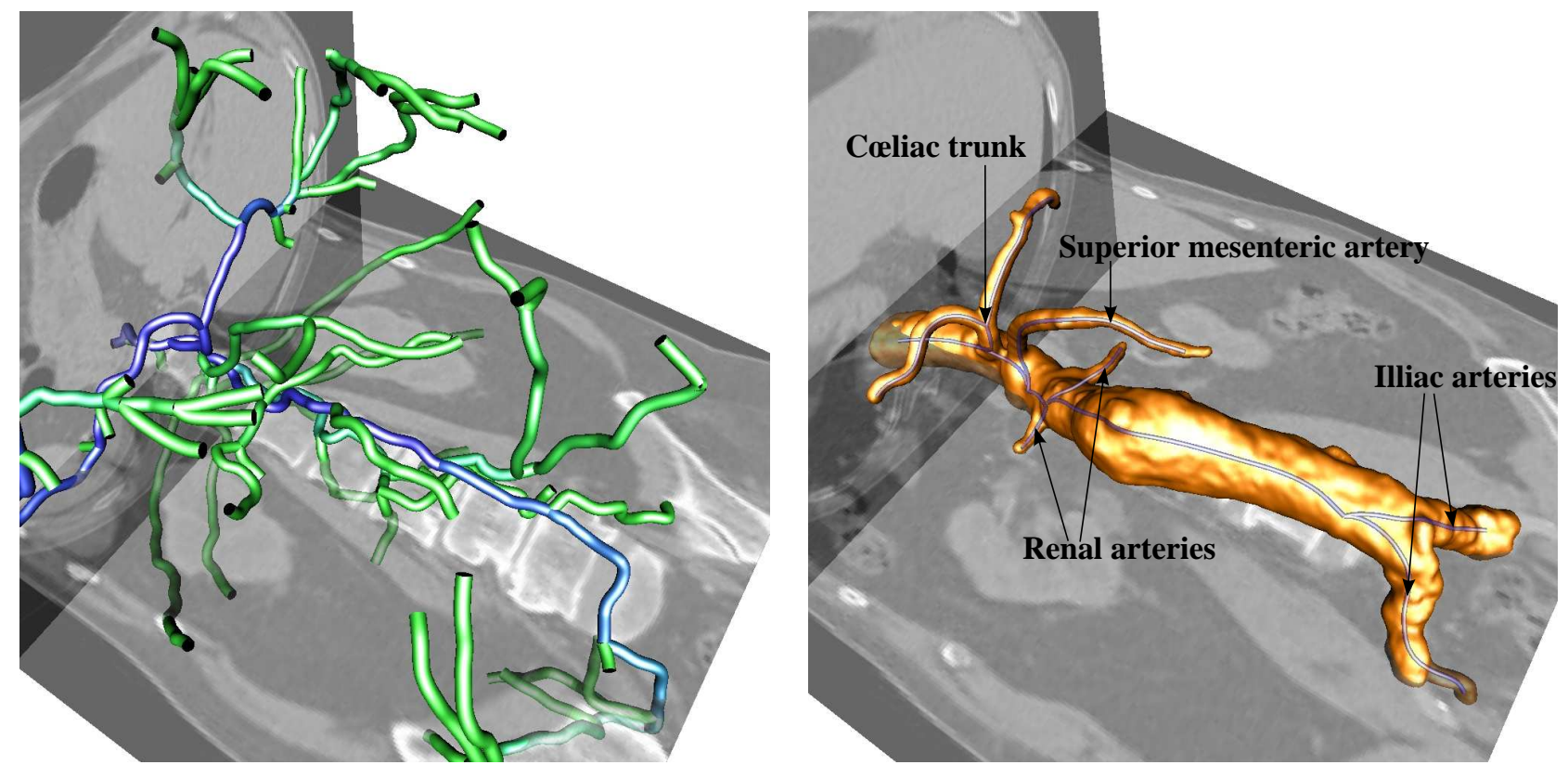

Figure 6. Tree after thresholding on voting score (left) and final tree with boundary surface (right) 

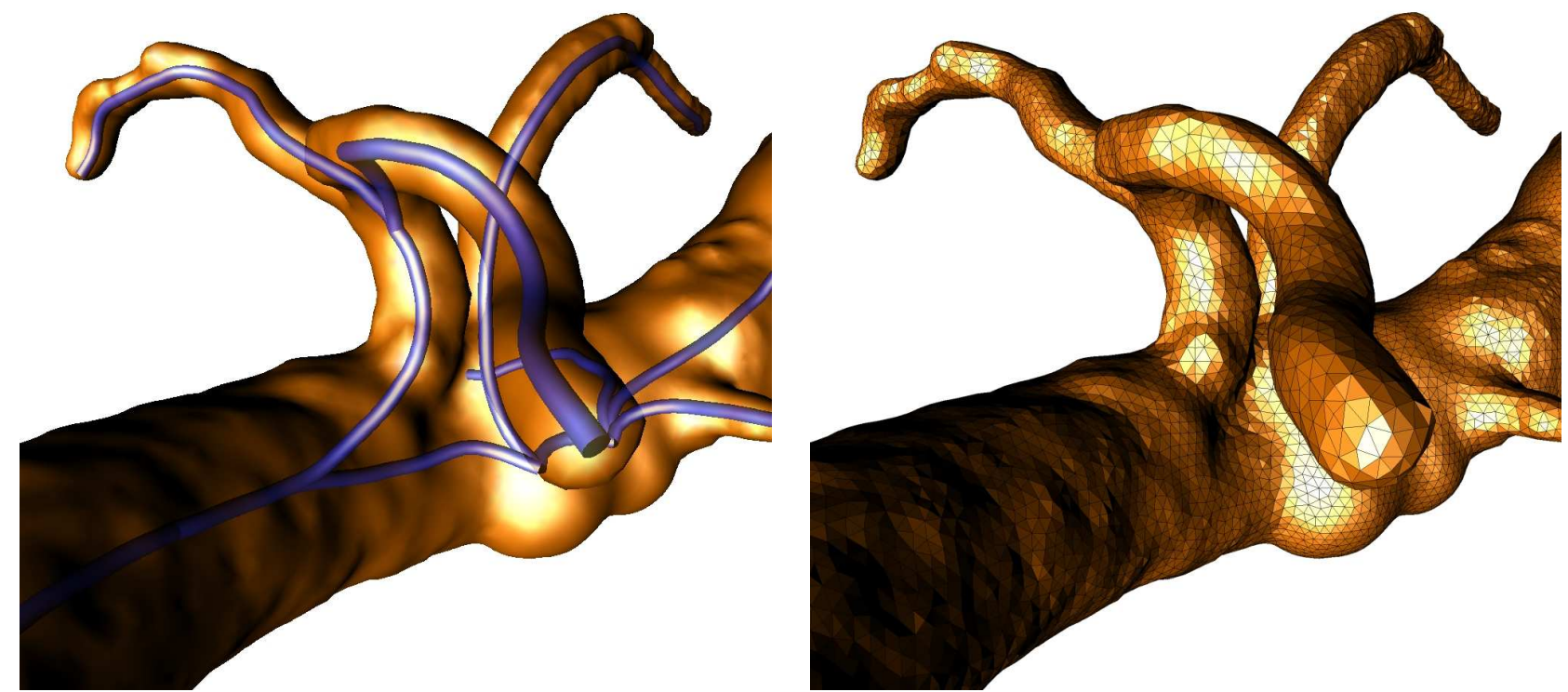

Figure 7. A close look at the boundary surface: smooth representation with centerlines (left) and triangulated representation (right)

of the cerebral vascular tree on 3D magnetic resonance angiography images. Medical Image Analysis, 5(3): 173-183, 2001.

[7] A. Frangi, W. Niessen, R. Hoogeveen, T. van Walsum, and M. Viergever. Model-based quantitation of 3D magnetic resonance angiographic images. IEEE Transactions on Medical Imaging, 18(10):946-956, 1999.

[8] M. Gülsün and H. Tek. Robust vessel tree modeling. In MICCAI, pages 602-611, New York, USA, 2008.

[9] A. Hilton, A. Stoddart, J. Illingworth, and T. Wandeatt. Marching triangles: range image fusion for complex object modeling. In ICIP, pages 381-384, Lausanne, Switzerland, 1996.

[10] A. Kaufman. Efficient algorithms for 3D scanconversion of parametric curves, surfaces, and volumes. Computer Graphics, 21(3):171-179, 1987.

[11] C. Kirbas and F. Quek. A review of vessel extraction techniques and algorithms. ACM Computing Surveys, 36(2):81-121, 2004.

[12] J. Lachaud and A. Montanvert. Deformable meshes with automated topology changes for coarse-to-fine three-dimensional surface extraction. Medical Image Analysis, 3(2):187-207, 1999.

[13] I. Laptev, H. Mayer, T. Lindeberg, W. Eckstein, C. Steger, and A. Baumgartner. Automatic extraction of roads from aerial images based on scale space and snakes. Machine Vision and Applications, 12(1):2331, 2000.
[14] J. Lee, P. Beighley, E. Ritman, and N. Smith. Automatic segmentation of 3D micro-CT coronary vascular images. Medical Image Analysis, 11(6):630-647, 2007.

[15] H. Li and A. Yezzi. Vessels as 4-D curves: global minimal 4-D paths to extract 3-D tubular surfaces and centerlines. IEEE Transactions on Medical Imaging, 26(9):1213-1223, 2007.

[16] J. Mille, R. Boné, and L. Cohen. Region-based 2D deformable generalized cylinder for narrow structures segmentation. In ECCV, pages 392-404, Marseille, France, 2008.

[17] T. O'Donnell, T. Boult, X. Fang, and A. Gupta. The Extruded Generalized Cylinder: a deformable model for object recovery. In CVPR, pages 174-181, Seattle, USA, 1994.

[18] Y. Rouchdy and L. Cohen. Image segmentation by geodesic voting: application to the extraction of tree structures from confocal microscope images. In ICPR, Tampa, Florida, USA, 2008.

[19] J. Sethian. A fast marching level set method for monotonically advancing fronts. Proceedings of the $\mathrm{Na}$ tional Academy of Science, 93(4):1591-1595, 1996.

[20] P. Yim, J. Cebral, R. Mullick, H. Marcos, and P. Choyke. Vessel surface reconstruction with a tubular deformable model. IEEE Transactions on Medical Imaging, 20(12):1411-1421, 2001. 